\title{
Functional Organization of Primary Somatosensory Cortex Depends on the Focus of Attention
}

\author{
Christoph Braun,*,1 Monika Haug,* Katja Wiech,* Niels Birbaumer,*'† \\ Thomas Elbert, $\ddagger$ and Larry E. Roberts§

\begin{abstract}
*Institute of Medical Psychology and Behavioral Neurobiology, University of Tübingen, 72076 Tübingen, Germany; †Center of Cognitive Neuroscience, University of Trento, Italy; ‡Department of Psychology, University of Konstanz, Germany;
\end{abstract} \\ and §Department of Psychology, McMaster University, Hamilton, Ontario, Canada
}

\begin{abstract}
We used magnetic source imaging in human subjects to reveal within-subject variations of the homuncular hand representation within the primary somatosensory cortex modulated by attention. I n one condition subjects were trained to detect sequential leftward or rightward stimulus motion across the fingers of the left hand ("hand" condition) and in a different condition to detect stimulus motion at a specific finger on this hand ('finger" condition). Afferent input was controlled by applying exactly the same stimulus pattern to the digits in the two tasks. Segregation of the somatotopic hand representation (an increase in the distance between the representations of digits 2 and 5) was observed, commencing with the onset of practice, in the finger relative to the hand condition. Subsequent training in the hand and finger conditions with feedback for correctness did not modify segregation, indicating that segregation was a task effect and not a training effect. These findings indicate that the hand representation within the primary somatosensory cortex is not statically fixed but is dynamically modulated by top-down mechanisms to support task requirements. A greater capacity for modulation of the functional cortical organization was positively correlated with superior learning and task performance. 2002 Elsevier Science (USA)
\end{abstract}

\section{INTRODUCTION}

Recent evidence indicates that neurons in the primary sensory cortices express a wide range of response properties that are not adequately described by the concept of a fixed receptive field established by early experience (Gilbert, 1998; Moore et al., 1999). For example, over a few seconds or less rat barrel neurons sharpen or broaden their tuning response to stimula-

\footnotetext{
${ }^{1}$ To whom reprint requests should be addressed at MEG-Center, University of Tübingen, Otfried-Müller-Strasse 47, 72076 Tübingen, Germany. E-mail: christoph.braun@uni-tuebingen.de.
}

tion of the vibrissae depending on the frequency of whisker deflection and on the spatial and temporal properties of patterned stimulation (Brumberg et al., 1996; Moore and Nelson, 1998; Sheth et al., 1998). When continued for hours to days, temporally coincident stimulation of adjacent whiskers leads to expansion of the receptive fields of neurons in all layers of primary somatosensory cortex (SI) (Armstrong-J ames et al., 1994; Diamond et al., 1994). These dynamics appear to be mediated by lateral connections that alter the tuning properties of neurons depending on the behavioral relevance and the intensity, pattern, or temporal coincidence of the stimuli (Moore et al., 1999). Similarly, the response of neurons in primary visual cortex to stimuli centered in their receptive fields is modulated by flanking stimuli presented concurrently outside the receptive field, particularly when attention is called to the flankers by the task (Crist et al., 2001; I to and Gilbert, 1999; M otter, 1993). In order to accommodate these findings it has become convenient to distinguish between the "classical" receptive field of a neuron which characterizes its response to simple transient stimulation and multiple "subthreshold" receptive fields which represent variations in the response of the cell that depend on the pattern of stimulation or on modulation of tuning dynamics by top-down mechanisms that reflect the information processing requirements of a task (Gilbert, 1998; Moore et al., 1999).

These findings have implications for the dynamics of representational maps of sensory surfaces which are established in the primary sensory cortices by early experience. Although extensive research in the past decade has shown that these maps can be modified in the adult brain by behavioral training over varying time scales (Buonomano and Merzenich, 1998), representational maps should also change dynamically with stimulus context and/or top-down attentional factors, insofar as maps reflect the tuning properties of the neurons that comprise them. In the present experi- 
ment we used neuromagnetic imaging in human subjects to investigate whether the homuncular organization of the hand area in primary somatosensory cortex is dynamically modulated by the attentional requirements of a task, when other task features are held constant. Subjects were required to detect the direction of stimulus motion (leftward or rightward) either across the digits of the left hand or at a single digit on the same hand. Task properties and stimulus pattern were identical in the two conditions, except for the requirement that attention be allocated differently to the digits on the two tasks. Under these circumstances differences in homuncular organization cannot arise from communication among sensory neurons receiving different stimulus input, but must instead reflect topdown modulation of the somatosensory representation by regions of the brain where task structure is coded.

\section{METHODS}

\section{Subjects}

Twelve right-handed, healthy subjects (mean age 30.5 years, range 25- 42 years, 6 females and 6 males) participated after having given informed consent.

\section{Procedure and Task}

For each subject somatosensory evoked magnetic fields were measured twice using a 151-channel whole-head magnetoencephaolgraph (CTF Systems, Vancouver, Canada). The first magnetoencephalographic (MEG) measurement was taken in a test session administered before subjects received training on the motion detection task, and the second MEG measurement was taken in a concluding test session administered after a minimum of three training sessions had been completed.

All sessions (test and training) were performed on separate days. In each session tactile stimuli separated by intervals of $250 \mathrm{~ms}$ were applied to the index (d2), middle (d3), ring (d4), or little (d5) fingers of the left hand. Each stimulus was delivered by a plastic tongue $2.5 \mathrm{~mm}$ wide that was swept across the finger either from right to left or from left to right by a pneumatically driven stimulator (Fig. 1). The direction of the sweep across each fingertip was varied randomly among the digits within the trial. However, within the trial neighboring fingers were stimulated in a consecutive sequence that could be perceived as moving from a randomly determined starting finger either leftward or rightward across the hand. In the example shown in Fig. 1 stimulation was applied first to d3 $(0 \mathrm{~ms})$ and subsequently to $\mathrm{d} 4, \mathrm{~d} 5$, and then $\mathrm{d} 2$ at 250-ms intervals, corresponding to leftward movement across the hand. On other trials (determined randomly and not shown in Fig. 1) movement across the hand was re- versed, again from a random starting digit (for example, d2, d5, d4, and d3, or d5, d4, d3, and d2, both examples rightward hand sweeps). Digit 1 (the thumb) was omitted from the task because hand position did not afford comfortable placement of this digit on stimulation probes (see Fig. 2b). In different training blocks, subjects had to detect either the direction of apparent motion across the hand ("hand" condition) or the direction of the stimulus at the $\mathrm{d} 2$ digit ("finger" condition). Subjects received visual feedback for the accuracy of their discriminative responses (correct/incorrect, plus a running total of the percentage of trials correct) on a trial-by-trial basis during training sessions, but not during test sessions. In all training and test sessions the hand and finger conditions were applied twice, in a counterbalanced order.

Each of the two repetitions applied in the hand and finger conditions within each session contained 400 trials. Trials were of 1.5-s duration and were initiated $1.5 \mathrm{~s}$ after the subject's discriminative response. Each finger was stimulated once during a trial; the time required to complete a sweep across a single finger was $53 \pm 16 \mathrm{~ms}$. On half the trials the fingers were stimulated in a rightward hand sequence and on the remaining trials in a leftward hand sequence (trial types randomly intermixed), with the direction of stimulus motion across individual fingers randomized between left and right sweeps. Subjects indicated the perceived stimulus direction (hand or finger condition) by pressing one of two buttons with the index finger of their right (nonstimulated) hand during training sessions or during neuromagnetic testing by interrupting one of two photocells with this finger.

\section{Magnetic Source I maging}

Somatotopic representations of digits 2-5 were determined by applying magnetic source imaging to MEG measurements taken during the test sessions. The MEG was digitized at $625 \mathrm{~Hz}$ using a low-pass antialiasing filter of $208 \mathrm{~Hz}$ (open high-pass filter). The continuous MEG record on each trial was epoched into 500-ms windows containing the neuromagnetic response to each finger stimulation. Epochs containing eye movement artifacts exceeding $70 \mu \mathrm{V}$ in the electrooculogram were rejected. The mean rejection rate across subjects was $11.8 \%$. E pochs were then averaged across trials for finger type. The first prominent magnetic field component, the M60 (see Fig. 2a), which is evoked in the contralateral hemisphere 50-60 ms after tactile stimulation, was modeled by an equivalent current dipole using an individualized spherical head model fitted to the 3D digitized head surface of each subject. The generator of the M 60 component has been localized to neural sources in cortical area $3 \mathrm{~b}$ of SI by several previous studies (Cheyne et al., 1998; Elbert et al., 1995; Hari et al., 1993; Lütkenhöner et al., 1995). If 
present, ipsilateral activity (Fig. 2b) or residual eye activity was modeled by an extra source that was excluded from further analysis. In each subject the dipole model explained $90 \%$ or more of the variance of the measured magnetic field. Changes in the functional organization of digit representations were quantified using the distance al ong the central sulcus between the dipole locations of $\mathrm{d} 2$ and $\mathrm{d} 5(\Delta \vartheta$; for the definition of the polar coordinate system see Fig. 2c). The strength of cortical activation evoked by finger stimulation was evaluated using dipole moment.

\section{Statistical Analyses}

Behavioral performance was assessed by a threeway analysis of variance (ANOVA) including the within-subject factors Condition (hand and finger), Sessions (test session 1, training sessions $1-3$, and test session 2), and Repetition (first and second repetitions within sessions). Individual comparisons were evaluated within the ANOVA by post hoc $F$ ratios. These analyses were applied to detection accuracy (percentage correct) and reaction time. Detection accuracy was evaluated further by contrasting the test sessions given before and after training with a sign test. Because magnetic source imaging was conducted only on the test sessions, MEG data were evaluated by a four-way ANOVA including the factors Condition (hand and finger), Session (before/after test sessions), Repetition (first and second repetitions within session), and an additional factor Digit. Error probabilities for factors involving more than two levels were corrected for inhomogeneities in variance by the Greenhouse-Geisser procedure.

\section{RESULTS}

\section{Behavioral Performance}

All subjects improved at discrimination accuracy in the hand and finger conditions when the two MEG test sessions without feedback were compared before and after training (mean accuracy of 54.4 and $75.9 \%$, respectively, on these two sessions, $\mathrm{P}<0.0001$, sign test). I mprovements were evident between successive training sessions up to and including the third training session (minimum $F(1,11)=5.50, P=0.035$, between the second and third training sessions), after which only a small decrement averaging $-4.8 \%$ occurred when feedback was removed in the concluding test session $(F(1,11)=4.78, P=0.047)$. Discrimination accuracy improved between the first $(70.1 \pm 1.4 \%)$ and second $(71.6 \pm 1.4 \%)$ replications within sessions $(F(1,11)=5.94, P=0.033)$. No main effects or interactions attributable to condition (hand or finger) were observed, indicating that discrimination accuracy was comparable in the two tasks (overall mean accuracy was $71.6 \pm 1.5$ and $70.1 \pm 1.4 \%$ in the hand and finger conditions, respectively). Reaction times were longer in the hand condition (1213 $\pm 16 \mathrm{~ms}$, measured from the second stimulus which signaled motion direction) than in the finger condition $(990 \pm 17 \mathrm{~ms}, \mathrm{~F}(1,11)=100.5$, $P=0.0001)$, because in the hand condition subjects had an opportunity to sample additional stimuli before making their discriminative decisions. Subjects re sponded more quickly in the second (1016.8 $\pm 17.4 \mathrm{~ms})$ than in the first $(1084.0 \pm 17.6 \mathrm{~ms})$ repetition within sessions $(F(1,11)=46.12, P<0.0001)$.

\section{Somatotopic Representations}

A strongly dipolar neuromagnetic response was observed in the contralateral hemisphere 50-60 ms following stimulation of each finger in all subjects in the hand and finger conditions (the M60, Fig. 2a). We evaluated the effect of task condition on the somatotopic representation by using the current dipoles that were fitted to this component for each finger. The spatial location parameters polar angle $\vartheta$ (representing the center of cortical activation along the central sulcus), the azimuth angle $\varphi$ (representing location in the axial $x / y$ plane), and radial eccentricity $r$ were calculated (see Fig. 2c). No effects were found in radial eccentricity $(r)$ or source location within the $x / y$ plane $(\varphi)$. However, a main effect of digit was found for polar angle $(F(2,22)=23.19, \varepsilon=0.919, P=0.0001)$, computed in this case with digits 2,3 , and 4 referenced to d5. Figure 3 shows that the relative positions of sources modeling activations for digits 2, 3, and 4 were shifted along the central sulcus with respect to sources for digit 5 in both conditions, in accordance with Penfield's somatotopy. An interaction of digit with task condition was also found for polar angle $(F(2,22)=$ 7.157, $\varepsilon=0.78, \mathrm{P}=0.004$ ) and can be seen in Fig. 3. A larger distance was observed between digits 5 and 2 $\left(\Delta \vartheta_{\mathrm{d} 5-\mathrm{d} 2}\right)$ in the finger condition than in the hand condition (post hoc $F(1,11)=26.07, P=0.001$ ), with no effect of task condition for distances between digit 5 and digits 3 and $4\left(\Delta \vartheta_{\mathrm{d} 5-\mathrm{d} 3}\right.$ and $\Delta \vartheta_{\mathrm{d} 5-\mathrm{d} 4}$, post hoc $\mathrm{F}$ 's $\left.<1\right)$. When we rereferenced polar angle to digit 2, the angle differences $\Delta \vartheta_{\mathrm{d} 2-\mathrm{d} 3}$ and $\Delta \vartheta_{\mathrm{d} 2-\mathrm{d} 4}$ were found to be significantly larger in the finger condition than in the hand condition $(F(1,11)=13.16, P=0.004)$ and more so for digit $4\left(\Delta \vartheta_{\mathrm{d} 2-\mathrm{d} 4}\right)$ than for digit $3\left(\Delta \vartheta_{\mathrm{d} 2-\mathrm{d} 3}, \mathrm{~F}(1,11)=5.85\right.$, $P=0.0341)$. These results indicate that the cortical representation for digit 2 was segregated from the representations for digits 3, 4, and 5 in the finger condition, whereas the location of sources for digits 3, 4, and 5 remained much the same between conditions. The positions of dipoles modeling digits 2 and 5 and the polar angle between them $\left(\Delta \vartheta_{\mathrm{d} 5-\mathrm{d} 2}\right)$ are cor egistered on the MRI of a representative subject for the finger and hand conditions in Fig. 2C, to illustrate the location of the cortical sources of the M60 event in the somatosen- 
a

Preparation Stimulation
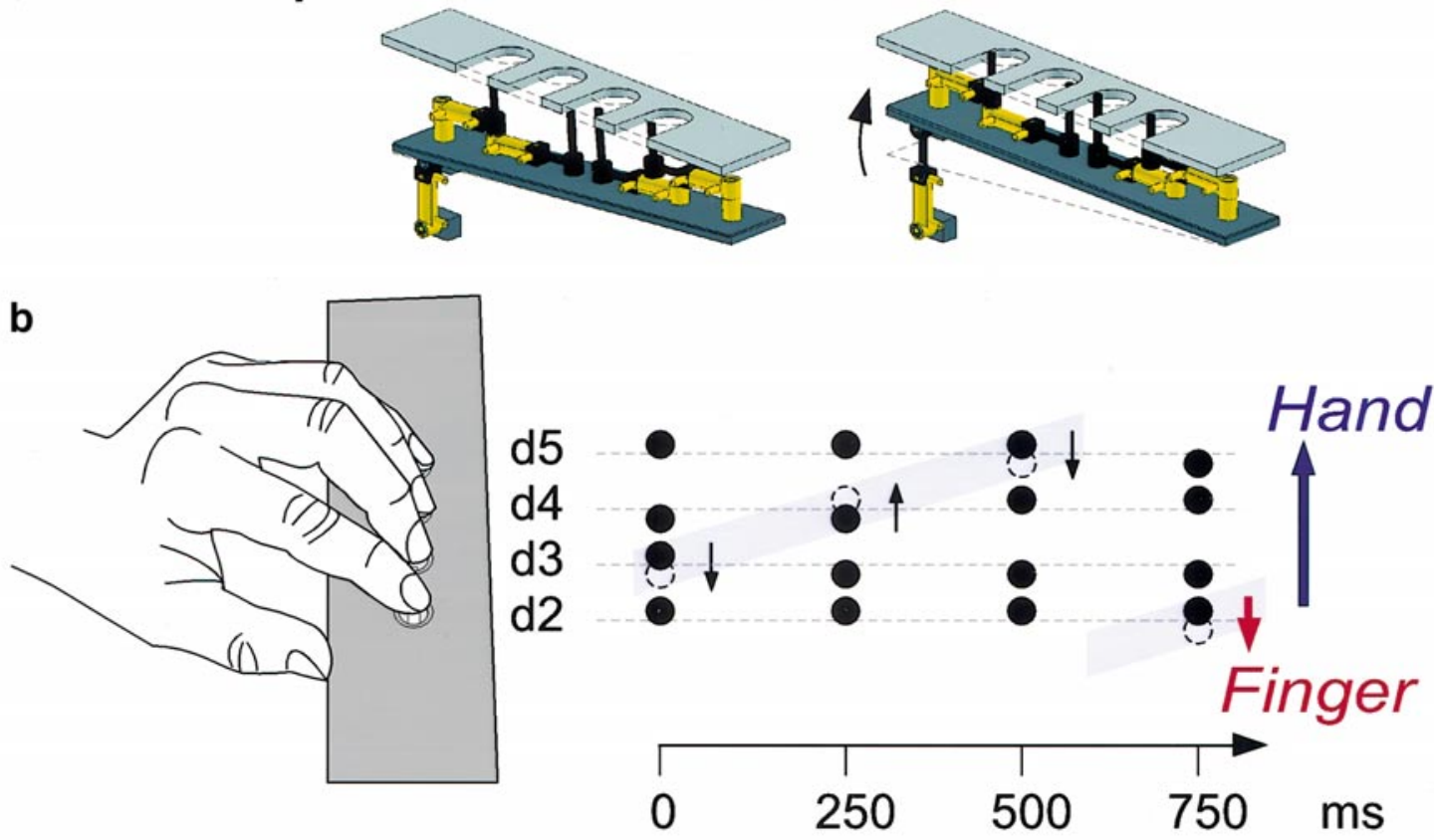

c Hand

Finger

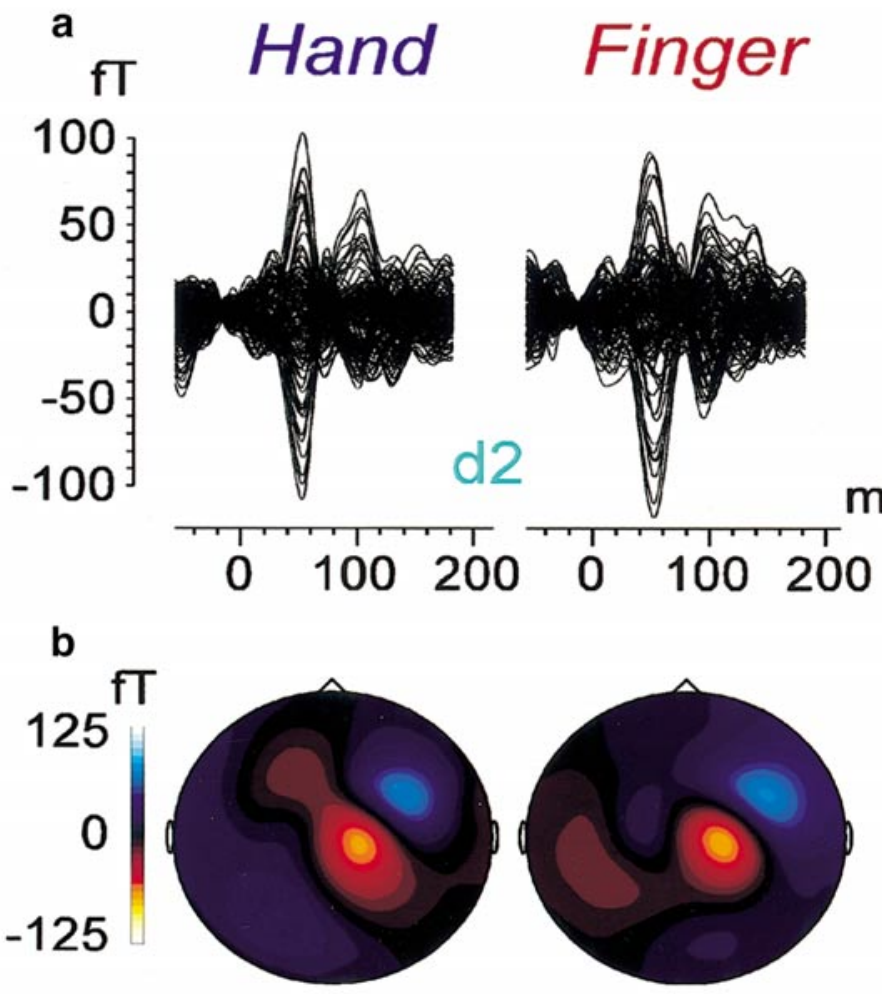

2

FIG. 1. Tactile stimulator and stimulus procedure. The stimulation device is shown in (a) and hand position and a typical stimulus sequence in (b). (a) During preparation the stimulator platform was retracted so that probes could be moved to their starting positions without contacting the fingertips. During the stimulation phase the platform was raised such that the probe tips were positioned to the side of each finger prior to the sweep. (b) The starting positions for each probe are shown at $0 \mathrm{~ms}$ (filled circles) and correspond with those illustrated for stimulation in (a). At this time point d3 was swept rightward (small arrow) to the end position indicated by the dotted circle. Subsequently d4 was swept leftward at $250 \mathrm{~ms}$, d5 rightward at $500 \mathrm{~ms}$, and d2 rightward at $750 \mathrm{~ms}$ (end of trial). In the finger condition the subject's task 
sory cortex and the polar angle shift with task condition.

We also examined whether changes may have been induced in the somatosensory representations by practice at detection of stimulus motion during the training sessions. Differences in polar angle between the finger and hand conditions did not change significantly for any digit between the MEG test sessions administered before and after training (all F's involving session $<1$ ), indicating that polar angle was affected by the task manipulation and not by practice. However, a significant correlation was found relating the difference in polar angle for the d2- d5 representation $\left(\Delta \vartheta_{\mathrm{d} 5-\mathrm{d} 2}\right)$ be tween the finger and hand tasks and improvement in discrimination accuracy on the two tasks from the initial to the closing test session, $r=0.60, t(11)=2.38$, $\mathrm{P}=0.039$. This relationship revealed that subjects showing the largest shifts (attentional modulation) of d5- d 2 distance between the finger and hand conditions of the first session improved most at discrimination on the two tasks.

Dipole moment was evaluated by the same analyses applied to polar angle. An effect of condition was found $(F(1,11)=4.93, P=0.0484)$ which was attributable to larger dipole moments occurring in the hand (17.8 \pm $0.9 \mathrm{nAm})$ than in the finger $(15.3 \pm 0.8 \mathrm{nAm})$ task. A main effect attributable to finger was also found $(F(3,33)=2.88, P=0.051)$ owing to a smaller dipole moment for d5 (14.1 nAm) compared to the other digits (17.4 nAm, averaged across d2- d4). Main effects and interactions attributable to before/after test sessions did not reach significance for dipole moment. Thus, as was true for polar angle, effects of the task manipulation on dipole moment were not altered by practice.

A final analysis evaluated the adequacy of the dipole model for representing the somatotopic organization of the digits. Residual variances averaged 4.0 and $4.9 \%$ in the hand and finger conditions, respectively, and did not exceed $10.4 \%$ for any subject or finger. These residuals are within conventional limits ( $<10 \%)$ for measuring somatotopic representations of the digits (EI-

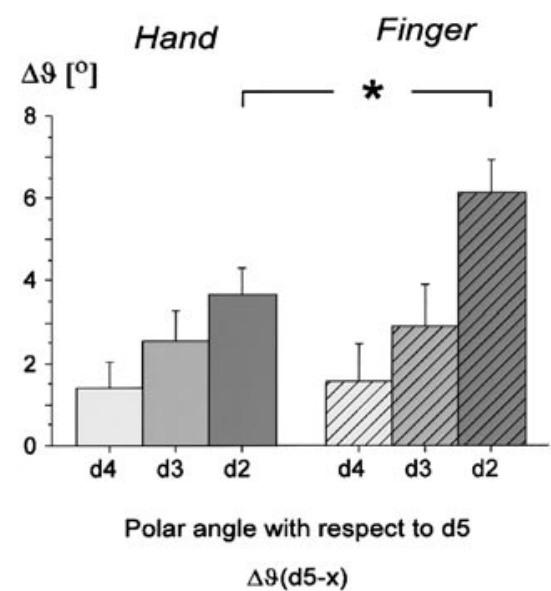

FIG. 3. Mean differences in polar angle $\Delta \vartheta$ between cortical representations for digits 2, 3, and 4 referred to digit 5, for the finger and hand conditions. $\Delta \vartheta$ reflects shifts in the centers of cortical activation for the digits along the central sulcus (see Fig. $2 \mathrm{C})$. The main effect of digits $(P=0.0001)$ reflects the homuncular organization of the fingers, with sources for d5 most distant from sources for $\mathrm{d} 2$ and the other digits lying in between. The interaction of digits with task $(P=0.004)$ was due to a larger $\Delta \vartheta$ $(d 5-d 2)$ in the finger compared to the hand condition $(P=0.001$, asterisk) and reflects modulation of the homuncular representation by task requirements.

bert et al., 1995; Hari et al., 1993; Lütkenhöner et al., 1995). Effects of finger $(F(3,33)=10.8, P<0.0001)$ and condition $(F(1,11)=8.10, P=0.016)$ were found for residual variance which reflected larger residuals occurring for digit 5, particularly in the finger condition. These effects on residual variance mirror those on dipole moment and indicate slightly better dipole fits when signal strength is large. The effect of the task manipulation (hand minus finger) on polar angle for $\mathrm{d} 2$ (the segregated finger, referred to d5) was unrelated to differences in residual variance for $\mathrm{d} 2$ and $\mathrm{d} 5$ between the hand and finger conditions ( $r=0.269$ and 0.211 for d2 and d5, respectively, $P>0.40$ in both cases). 


\section{DISCUSSION}

The subjects of this study were required to detect the direction of motion of a tactile stimulus either across the finger tips of the left hand (hand condition) or across a single digit of this hand (finger condition) when identical stimulation was delivered to the digits. This procedure was designed to alter the subject's focus of attention (global "hand" versus local "finger") while holding afferent input to the somatosensory cortex constant. We found that the cortical representation for digit 2 was segregated from that of digits 3-5 in the finger relative to the hand condition by the differing attentional requirements of these tasks. Dipole moment was also larger for the hand compared to the finger task, which is consistent with a more integrated finger representation occurring in the hand condition. It is unlikely that task difficulty contributed to these effects, because discrimination performance was comparable in the hand and finger tasks. Variability in the goodness of fit of our dipole model to the M60 magnetic field pattern also did not appear to have been a contributing factor, because small effects of task on residual variance reflecting signal strength did not correlate with task effects on polar angle. Because we modeled digit representations by point-like current dipoles, we cannot determine whether digits 3-5 were represented by different neurons in the finger and hand conditions or whether processes such as surround inhibition or coactivation of additional sources by attentional mechanisms modulated the neural representations so as to shift the center of activation observed for each digit in the two conditions. Regardless of which of these interpretations is accepted, cortical dynamics appear to have been modulated by top-down mechanisms, because the somatosensory cortex received the same afferent input during the hand and finger tasks.

Current dipoles fitted to the M60 component of somatosensory responses evoked by tactile stimuli have been localized to area 3b of SI by several neuromagnetic studies of somatotopy (Cheyne et al., 1998; El bert et al., 1995; Hari et al., 1993; Lütkenhöner et al., 1995). Our source localizations were consistent with these earlier findings. Because the M60 component preceded the behavioral responses of our subjects by several hundred milliseconds (response latencies were typically >900 ms), brain activity arising from the motor response could not have contributed to the findings reported here. It is also unlikely that differential modulation of secondary somatosensory cortex (SII) by attention (Mima et al., 1998; Backes et al., 2000) may have influenced our M60 dipole fits in the hand and finger conditions. When somatosensory magnetic fields are induced by haptic/tactile (touch) stimuli (the type of stimulation used in our study), the first major cortical response detected by MEG occurs in area $3 \mathrm{~b}$ of $\mathrm{SI}$ (for a review see E Ibert, 1998). Only when electrical or painful stimuli are used does activation of SII become prominent in MEG recordings (Elbert et al., 1995; Hari et al., 1993; Hari and IImoniemi, 1986; Howland et al., 1995; Huttunen et al., 1986; Kitamura et al., 1995), and even then SII responses are not reliably present in all subjects until 100 ms (Elbert et al., 1995; Hari et al., 1993), which is well after the M60 has reached its completion (Fig. 2a). Evidence reported by Karhu and Tesche (1999) suggests that SII responses may appear neuromagnetically with a latency prior to $60 \mathrm{~ms}$ in a subset of subjects when $2-\mathrm{Hz}$ trains of el ectrical stimuli are applied to a mixed nerve (the median nerve). When induced by electrical stimuli, these early SII responses appeared bilaterally and were distinguishable from contralateral SI M60 activations by their magnetic field orientation (Karhu and Tesche, 1999).

Previous studies with human subjects have shown that somatotopic representations can be altered by manipulating the temporal or spatial properties of stimuli delivered to the somatosensory cortex. For example, it has been reported that d1- d3 distances expand when patterned stimulation is delivered to digits 1,3 , and 5 compared to when these digits are stimulated in a random order (Braun et al., 2000a) and that d1-d5 distances expand when the intervening digits 2,3 , and 4 are anesthetized (Buchner et al., 1995, 1999) or when fingers conjoined by syndactly are separated by surgery (Mogilner et al., 1993). In addition, the strength of the cortical representation when multiple digits are stimulated concurrently is less than the sum of their individual representations (Liu et al., 2000), which suggests that digit representations are tuned by reciprocal interactions in agreement with findings reported by Mirabella et al. (2001) for rat barrel neurons. Somatosensory interactions that depend on stimulus pattern are believed to be mediated by excitatory and inhibitory connections among cortical columns tuned to different digits in the homuncular representation (Moore et al., 1999). However, in order to demonstrate attentional modulation within the homunculus, stimulus procedure must remain constant while the requirements for processing tactile information are manipulated by task procedure. In previous experiments reporting effects of attention on somatotopic organization, attention has been deployed either to task stimuli that activate different somatosensory cortices (e.g., count probes presented to the right or the left hand, Noppeney et al., 1999) or to stimuli that differ in their intensity, frequency, or pattern and thus convey different input to the somatosensory cortex during the attention conditions (e.g., count oddball deviants in different stimulus arrangements, Mima et al., 1998; Iguchi et al., 2001). In contrast, we used tasks that required processing of the same stimulus pattern in different ways. Because the stimulus pattern was identical for the two tasks, attention was deployed within 
the same somatotopic representation, and modulation of this representation was seen.

In addition to stimulus pattern and attention, repetitive experience with behaviorally relevant tactile stimulation delivered by procedures such as whisker pairing (Armstrong-J ames et al., 1994; Diamond et al., 1994), discriminative conditioning (J enkins et al., 1990), or discrimination among stimuli presented synchronously (fusion) or asynchronously (segregation) to multiple digits (Wang et al., 1995; Sterr et al., 1998; Braun et al., 2000b; Liu et al., 2000) can also alter the tuning of neurons in the somatosensory cortex. Because modifications induced by behavioral training are progressive and persist beyond the period in which training is delivered, neuroplastic processes are likely involved that modify synaptic connections within at least a selected region of the dendritic arbor (Buonomano and Merzenich, 1998). In the present study subjects improved their detection of stimulus motion during training in the hand and finger conditions, and their improved skill persisted in the concluding test session on which feedback was removed, with only a small decrement in discrimination accuracy in this session compared to the preceding session where feedback was provided. Attentional modulation of the somatosensory representation (which was fully expressed at the outset of training) appears to have contributed to learning, because behavioral improvement was greatest for subjects for whom the d2 representation was most segregated from the remaining digits by the task manipulation.

Our findings are consistent with animal studies showing that the tuning of sensory neurons in $\mathrm{V} 1$ or rat barrel fields is not statically fixed but is dynamically modulated by top-down influences from other regions of the brain that convey information about training context and/or attentional requirements (Gilbert, 1998). Sensory neurons appear to multiplex (Crist et al., 2001) their functions among multiple subthreshold inputs in accordance with these variables. How modulation is achieved is an emerging problem for neuroscience research. It would appear that for top-down effects of attention and context to be expressed, higherorder representations of lower-level sensory features must exist, called "polymaps" by Swindale (2000) for the visual system, that can modulate activity in the primary somatosensory cortex according to task demands. Such maps may be continuously formed and modified by experience through neuroplastic mechanisms that are gated by attention. Our findings also call for caution in ascribing the effects of behavioral training to "cortical remodeling" by neuroplastic processes which produce persisting changes in synaptic efficacy. Because a homunculus can have different appearances depending on stimulus pattern and task requirements, the observation of two different views of a homunculus does not imply that remodeling has oc- curred, unless task variables and behavioral context have been well controlled.

\section{ACKNO WLEDGMENTS}

We thank Mr. Wolfgang Kern for constructing the stimulation device. This research was supported by the Deutsche Forschungsgemeinschaft (SFB 550/C6). The participation of LER was assisted by grants from the German American Academic Council and the Canadian Institutes of Health Research.

\section{REFERENCES}

Armstrong-J ames, M., Diamond, M. E., and Ebner, F. F. 1994. An innocuous bias in whisker use in adult rats modifies receptive fields of barrel cortex neurons. J . Neurosci. 14: 6978-6991.

Backes, W. H., Mess, W. H., van Kranen-Mastenbroeck, V., and Reulen, J. P. H. 2000. Somatosensory cortex responses to median nerve stimulation: $\mathrm{fMRI}$ effects of current amplitude and selective attention. Clin. Neurophysiol. 111: 1738-1744.

Braun, C., Wilms, A., Schweizer, R., Godde, B., Preissl, H., and Birbaumer, N. 2000a. Activity patterns of human somatosensory cortex adapt dynamically to stimulus properties. NeuroReport 11: 2977-2980.

Braun, C., Schweizer, R., Elbert, T., Birbaumer, N., and Taub, E. 2000b. Differential activation in somatosensory cortex for different discrimination tasks. J. Neurosci. 20: 446- 450.

Brumberg, J . C., Pinto, D. J ., and Simons, D. J . 1996. Spatial gradients and inhibitory summation in the rat whisker barrel system. J . Neurophysiol. 76: 130-140.

Buchner, H., Reinartz, U., Waberski, T. D., Gobbele, R., N oppeney, U., and Scherg, M. 1999. Sustained attention modulates the immediate effect of de-afferentation on the cortical representation of the digits: Source localization of somatosensory evoked potentals in humans. Neurosci. Lett. 260: 57-60.

Buchner, H., Kauert, C., and Radermacher, I. 1995. Short-term changes of finger representation at the somatosensory cortex in humans. Neurosci. Lett. 198: 57-59.

Buonomano, D. V., and Merzenich, M. M. 1998. Cortical plasticity: From synapses to maps. Annu. Rev. Neurosci. 21: 149-186.

Cheyne, D., Roberts, L. E., Gaetz, W., Bosnyak, D. J ., Nahmias, C., Christoforou, N., and Weinberg, H. 1998. Somatotopic organization of human somatosensory cortex: A comparison of EEG, MEG and fMRI methods. In Brain Topography Today (Y. Koga, K. Nagata, and K. Hirata, Eds.), pp. 76- 81. Elsevier Excerpta Medica International Congress Series, Amsterdam.

Crist, R. E., Li, W., and Gilbert, C. D. 2001. Learning to see: Experience and attention in primary visual cortex. Nat. Neurosci. 4: 519-525.

Diamond, M. E., Huang, W., and Ebner, F. F. 1994. Laminar comparison of somatosensory cortical plasticity. Science 265: 18851888.

Elbert, T., J unghöfer, M., Scholz, B., and Schneider, S. 1995. Separation of overlapping neuronal sources in the first and second somatosensory cortices. Brain Topogr. 7: 275-282.

Elbert, T. 1998. Neuromagnetism. In Magnetism in Medicine (W. Andrä and H. Nowak, Eds.), pp. 190-262. Wiley, New York.

Gilbert, C. D. 1998. Adult cortical dynamics. Physiol. Rev. 78: 467485.

Hari, R., and IImoniemi, R. J . 1986. Cerebral magnetic fields. Crit. Rev. Biomed. Eng. 14: 93-126.

Hari, R., Karhu, J., Hamalainen, M., Knuutila, J., Salonen, O., Sams, M., and Vilkman, V. 1993. Functional organization of the 
human first and second somatosensory cortices: A neuromagnetic study. Eur. J . Neurosci. 5: 724-734.

Howland, E. W., Wakai, R. T., Mjaanes, B. A., Balog, J. P., and Cleeland, C. S. 1995. Whole head mapping of magnetic fields following painful electric finger shock. Cogn. Brain Res. 2: 165172.

Huttunen, J ., Kobal, G., Kaukoranta, E., and Hari, R. 1986. Cortical responses to painful CO2 stimulation of nasal mucosa; A magnetoencephalographic study in man. Electroencephalog. Clin. Neurophysiol. 64: 347-349.

I guchi, Y., Hoshi, Y., and Hashimoto, I. 2001. Selective spatial attention induces short-term plasticity in human somatosensory cortex. NeuroReport 12: 3133-3136.

Ito, M., and Gilbert, C. D. 1999. Attention modulates contextual influences in the primary visual cortex of alert monkeys. Neuron 22: 593- 604.

J enkins, W. M., Merzenich, M. M., Ochs, M. T., Allard, T., and Guic-Robles, E. 1990. Functional reorganization of primary somatosensory cortex in adult owl monkeys after behaviourally controlled tactile stimulation. J . Neurophysiol. 63: 82-104.

Karhu, J ., and Tesche, C. D. 1999. Simultaneous early processing of sensory input in human primary (SI) and secondary (SII) somatosensory cortices. J . Neurophysiol. 81: 2017-2025.

Kitamura, Y., Kakigi, R., Hoshiyama, M., Koyama, S., Shimojo, M., and Watanabe, S. 1995. Pain-related somatosensory evoked magnetic fields. Electroencephalogr. Clin. Neurophysiol. 95: 463- 474.

Liu, L. C., Gaetz, W. C., Bosnyak, D. J ., and Roberts, L. E. 2000. Evidence for fusion and segregation induced by $21-\mathrm{Hz}$ multipledigit stimulation in humans. NeuroReport 11: 2313-2318.

Lütkenhöner, B., Menninghaus, E., Steinstrater, O., Wienbruch, C., Gissler, H. M., and Elbert, T. 1995. Neuromagnetic source analysis using magnetic resonance images for the construction of source and volume conductor model. Brain Topogr. 7: 291-299.
Mima, T., Nagamine, T., Nakamura, K., and Shibasaki, H. 1998. Attention modulates both primary and secondary cortical activities in humans: A magnetoencephalographic study. J. Neurophysiol. 80: 2215-2221.

Mirabella, G., Battiston, S., and Diamond, M. E. 2001. Integration of multiple-whisker inputs in rat somatosensory cortex. Cereb. Cortex 11: 164-170.

Mogilner, A., Grossman, J . A., Ribary, U., J oliot, M., Volkmann, J ., Rapaport, D., Beasley, R. W., and Llinas, R. R. 1993. Somatosensory cortical plasticity in audlt humans revealed by magnetoencephalography. Proc. Nat. Acad. Sci. USA 90: 3593-3597.

Moore, C. I., Nelson, S. B., and Sur, M. 1999. Dynamics of neuronal processing in rat somatosensory cortex. Trends Neurosci. 22: 513520.

Moore, C. I., and Nelson, S. B. 1998. Spatio-temporal subthreshold receptive fields in the vibrissa representation of rat primary somatosensory cortex. J . Neurophysiol. 80: 2882-2892.

Motter, B. C. 1993. Focal attention produces spatially selective processing in visual cortical areas V1, V2, and V4 in the presence of competing stimuli. J . Neurophysiol. 70: 909-919.

Noppeney, U., Waberski, T. D., Gobbele, R., and Buchner, H. 1999. Spatial attention modulates the cortical somatosensory representation of the digits in humans. NeuroReport 10: 3137-3141.

Sheth, B. R., Moore, C. I., and Sur, M. 1998. Temporal modulation of spatial borders in rat barrel cortex. J . Neurophysiol. 79: 464-470.

Sterr, A., Müller, M. M., Elbert, T., Rockstroh, B., Pantev, C., and Taub, E. 1998. Perceptual correlates of use-dependent changes in cortical representation of the fingers in blind Braille readers. J . Neurosci. 18: 4417-4423.

Swindale, N. V. 2000. How many maps are there in Visual Cortex? Cereb. Cortex 10: 633-643.

Wang, X., Merzenich, M. M., Sameshima, K., and J enkins, W. M. 1995. Remodeling of hand representation in adult cortex determined by timing of tactile stimulation. Nature 378: 71-75. 